\title{
Un voyage dans les derniers cercles de l'Enfer : $L a$ casa de los náufragos (Boarding home) de Guillermo Rosales
}

Marie-Caroline Leroux

\section{OpenEdition}

Journals

Édition électronique

URL : https://journals.openedition.org/cher/12169

DOI : $10.4000 /$ cher. 12169

ISSN : 2803-5992

Éditeur

Presses universitaires de Strasbourg

Édition imprimée

Date de publication : 30 juin 2012

Pagination : 89-101

ISBN : 978-2-35410-046-9

ISSN : 1968-035X

Référence électronique

Marie-Caroline Leroux, « Un voyage dans les derniers cercles de l'Enfer : La casa de los náufragos

(Boarding home) de Guillermo Rosales », reCHERches [En ligne], 8 | 2012, mis en ligne le 21 février 2022, consulté le 23 février 2022. URL : http://journals.openedition.org/cher/12169 ; DOI : https://doi.org/ 10.4000/cher.12169

\section{cc) (1) $\$($ )}

Ce(tte) œuvre est mise à disposition selon les termes de la Licence Creative Commons Attribution -

Pas d'Utilisation Commerciale - Partage dans les Mêmes Conditions 4.0 International. 


\section{Un voyage dans les derniers cercles de l'Enfer: La casa de los náufragos (Boarding home) de Guillermo Rosales}

Marie-Caroline Leroux

Université de Limoges

$\ll \coprod \begin{gathered}\text { aissez toute espérance, vous qui entrez». L'avertissement de Dante } \\ \text { au visiteur des Enfers n'aurait pas déparé au fronton du boarding }\end{gathered}$ home floridien dans lequel échoue William Figueras, triste jumeau de papier de l'écrivain cubain Guillermo Rosales, comme lui écrivain, comme lui exilé, comme lui frappé de schizophrénie, comme lui, enfin, interné dans un de ces sordides hospices privés où la folie le dispute à la cruauté et à l'ordure.

Que l'on s'attache aux conditions dans lesquelles elle a été écrite, à sa teneur, à sa fortune ${ }^{1}$ ou au destin de son auteur, on éprouve d'emblée que nul aspect de l'œuvre n'échappe à l'implacable logique de la flétrissure. Aussi tenterait-on vainement de l'embrasser tout d'une pièce. On la prendra d'abord pour ce qu'elle est selon toute apparence - un simple roman -, pour constater que la sensation de faillite s'insinue dans les moindres replis de l'univers diégétique. William Figueras a seul en charge le récit, qu'il sature ad nauseam de son propre désabusement. Mais l'échec n'est pas circonscrit à la sphère de la conscience douloureuse du protagoniste, il se déploie dans une topie, celle de l'asile, où échouent crève-la-faim, déments et vieillards séniles. La figure du cercle dantesque se trouve répliquée et dilatée une première fois dans ce microcosme. Quant au troisième cercle, il est

1 Ce roman valut à Rosales une reconnaissance plus qu'éphémère. Primé dans un concours littéraire présidé par Octavio Paz, il fut publié dans de déplorables conditions, en 1987, et tomba très vite dans l'oubli. 
indépendant des précédents. C'est le macrocosme - Miami -, qui le dessine, et il n'englobe pas l'asile, qui lui est plutôt une honteuse excroissance et témoigne de l'impossibilité des sociétés contemporaines à voisiner avec leurs marges. Bien qu'il n'ait d'autre horizon géographique que Miami, il est clair que cet ailleurs incarne le monde aux yeux de William. Un monde en retrait duquel il se positionne avec un faux détachement et qui lui fait dire: «No soy un exiliado político. Soy un exiliado total» (Rosales 2003: 11).

Le boarding home, il le sait, est sa dernière escale. La contraction de son espace intime s'opère au seuil du roman, avec un saisissant effet d'apnée pour le lecteur, à qui tout sera donné sous la forme d'un présent narratif qui va fixer - figer - le temps et l'espace: «Miro al techo. Es un techo azul, descascarado, recorrido por minúsculas cucarachas carmelitas. Bien. Este es mi final» (20). Plutôt que de fuir l'air vicié de ce lieu abominable, de tenter de s'en déprendre, William s'abîme dans le spectacle qui s'y joue. La présence de l'asile est à ce point oppressante qu'on en oublierait presque les déambulations urbaines de William et cet autre espace que constitue Cuba. Un espace presque entièrement oblitéré par la distance et par le monstrueux dépaysement que sa nouvelle situation impose au protagoniste, au point que l'Ile ne semble plus avoir de consistance que virtuelle. Elle prend place, d'abord, dans le songe et le souvenir. Il faudrait ajouter: un souvenir rendu plus débile encore par sa qualité de mémoire d'un temps depuis longtemps révolu. Lorsque William évoque Cuba, ce n'est pas telle qu'elle se maintient hors de lui ni même telle qu'il l'a laissée: sa nostalgie va aux premières années de la Révolution. Car il a perdu Cuba loin en amont de l'exil, lorsque l'idéal en lequel il avait foi s'est trouvé dévoyé. Ce qui m'amène au mythe, ou plutôt, au détricotage du mythe, qui dote Cuba d'un surcroît de virtualité. L'effondrement du fantasme d'une révolution aux accents généreux n'est qu'un des deux visages du mythe cubain. Le second se constitue sur l'envers du premier et reprend les termes d'un discours confit dans la rhétorique du péril rouge. On se souviendra notamment de ces tracts signés «Cubanos Vengadores» et des vitupérations d'un des pensionnaires:

¡Eso!-grita-. ¡Diez comunistas muertos! ¡Cien es lo que hace falta! ¡Mil! ¡Un millón de comunistas muertos! Lo que hay que hacer es llenarse de cojones y arrasar. Primero México. Luego Panamá. Luego Venezuela y Nicaragua. Y luego limpiar a los Estados Unidos que están infestados de comunistas! (39)

William dresse l'une contre l'autre ces deux visions, interdisant par là-même que l'image de Cuba s'y sédimente: la légende dorée de la Révolution est irrémédiablement ternie par le désenchantement de William; quant à la 
légende noire, elle est convoquée dans des scènes où le ton de la farce le dispute à l'emphase. Il y aurait là matière à un long développement sur la mise en échec des idéologies, qui est un aspect non négligeable de l'œuvre, et participe du désarroi du personnage ${ }^{2}$.

Il ressort de tout cela que l'exil dont se réclame véritablement le protagoniste n'est pas arrachement à une patrie. Il relève bien plutôt de l'arrachement à l'être à soi. La défaite première, qui entraîne toutes les autres, est chez lui celle de la raison:

Hace veinte años terminé una novela en Cuba que contaba la historia de un romance $[. .$.$] . Los especialistas literarios del gobierno dijeron que mi novela$ era morbosa, pornográfica, y también irreverente, pues trataba al Partido Comunista con dureza. Luego me volví loco. Empecé a ver diablos en las paredes, comencé a oír voces que me insultaban, y dejé de escribir. Lo que me salía era espuma de perro rabioso. Un día, creyendo que un cambio de país me salvaría de la locura, salí de Cuba y llegué al gran país americano (13-14).

La mention que le personnage fait ici de la censure est suivie d'une brutale ellipse, qui nous autoriserait à voir dans ce «luego», bien plus qu'une mention circonstancielle, l'expression d'un rapport de cause à effet. $\mathrm{Ne}$ serait-il pas naturel d'imputer les troubles psychologiques de William au harcèlement que lui vaut ce premier roman? La suite du texte coupe court à toute conjecture en ce sens: la forme verbale «creyendo" dit assez la désillusion de William sur ce point: l'exil ne le guérira pas. On aurait donc grand tort de donner une portée pamphlétaire à ce texte et de le mettre sur le même plan que la lettre d'adieu d'un Reinaldo Arenas ${ }^{3}$ : Guillermo Rosales n’impute pas à la Révolution et à ses idéaux bafoués la responsabilité de son mal.

Si William dénigre de façon si systématique l'adhésion de ses compatriotes exilés à un modèle petit-bourgeois consumériste, on peut se demander si son attitude ne procède pas davantage d'une inadéquation à ce patron que d'un rejet viscéral et si le rejet du modèle n'est pas le fruit de son incapacité à s'y conformer. De là, sans doute, qu'il ne blâme pas complètement sa

2 J'ai convenu qu'on prendrait le texte comme un roman, mais il est difficile ici de ne pas être tenté de remplacer le terme de "personnage» par celui d' «auteur»

3 «Pongo fin a mi vida voluntariamente porque no puedo seguir trabajando. Ninguna de las personas que me rodean están comprometidas en esta decisión. Sólo hay un responsable: Fidel Castro. Los sufrimientos del exilio, las penas del destierro, la soledad $y$ las enfermedades que haya podido contraer en el destierro seguramente no las hubiera sufrido de haber vivido libre en mi país» (Arenas 1996: 343). 
tante Clotilde, qui le recueille à son arrivée à Miami et renonce après trois mois d'efforts à supporter la charge qu'il représente pour le placer dans le boarding home: "La entiendo", répète-t-il par deux fois. De là qu'il ne fasse montre que d'une animosité assez molle à l'endroit des autres membres de sa famille présents à l'aéroport. Et qu'il s'attache, avec un sens prononcé de l'autodérision, à poser sur lui-même l'impossible masque du triomphateur:

Creyeron que llegaría un futuro triunfador, un futuro comerciante, un futuro playboy; un futuro padre de familia que tendría una futura casa llena de hijos, y que iría los fines de semana a la playa y correría buenos carros y vestiría ropa de marca Jean Marc y Pierre Cardin; y lo que apareció en el aeropuerto el día de mi llegada fue un tipo enloquecido, casi sin dientes, flaco y asustado [...] (14).

William Figueras, en dépit de ses assauts contre le clinquant du rêve américain entretenu par la diaspora cubaine, est en somme un inadapté plus qu'un anarchiste. Quoi qu'il en soit, la schizophrénie de William le renvoie dos au monde, et la nature irréductible de cet échec, qui relève de la fatalité, lui interdit d'y puiser l'énergie du dépassement. Son admission au sein du boarding home signe à ses yeux la défaite ultime et s'accompagne d'un avachissement de tout son être. On éprouve d'autant plus violemment l'abdication de William qu'elle se trouve réfléchie dans chacun des résidents, prenant le tour d'une servilité quasi atavique chez le nain Napoleón, d'une opiniâtre volonté d'en finir chez le vieux Castaño, ou d'une soumission tremblante à la loi du mâle chez Francis.

La désarmante docilité avec laquelle le protagoniste s'abandonne dès le début au temps routinier de l'asile constituele signe le plus manifeste de son renoncement. Dans cet espace centripète, le désœuvrement qui accable les pensionnaires donne un relief extraordinaire aux nécessités les plus basiques: manger, dormir, déféquer. Les conflits qui agitent ce petit monde et lui sont autant de mornes soubresauts tournent immanquablement autour de ces préoccupations, auxquelles s'ajoute la satisfaction des besoins sexuels. Après l'improbable sursaut que constitue son échappée belle en compagnie de Francis, William réintègre l'asile, et le roman se referme sur le détail du menu du jour, comme pour signifier le resserrement définitif de ses horizons: "Afuera, la mulata Caridad llama a comer. Habrá pescado frío, arroz blanco y lentejas crudas» (100).

$\mathrm{Au}$ long de ces cent pages, Guillermo Rosales met donc sa prose sèche, acide, au service de l'examen presque anatomique du quotidien de William. Ainsi, le temps se dilate dans le récit minutieux de ses dégoûtantes ablutions : 
Desperté. Ha pasado un mes desde que estoy aquí, en el boarding home. Mi sábana sigue siendo la misma, mi funda también. La toalla que el señor Curbelo me diera el primer día, está ahora emporcada y húmeda y huele fuertemente a sudor. La tomo y me la echo al cuello. Voy al baño a asearme y a orinar. Llego al baño. Orino sobre una camisa a cuadros que algún loco ha metido en la taza. Luego me vuelvo al lavamanos y abro una de las llaves. $\mathrm{Me}$ froto la cara con agua fría. Me seco con la toalla emporcada (47).

Sa vie est rythmée par ces non-événements que sont les moments de repas ou de toilette. La relégation de William vaut pour une entrée dans le temps mort. Dans ce passage, l'état de sa serviette en deviendrait presque la mesure du temps écoulé. Il faut là rappeler qu'en dépit de la présence dans le texte de quelques balises temporelles, le lecteur est dans l'incapacité d'évaluer l'intervalle qui sépare l'arrivée de William et la ruine de ses espoirs.

Cette impression d'un temps coagulé tient à plusieurs éléments, au nombre desquels on trouve la narcolepsie dont semble frappé William. Ses assoupissements répétés nient à tel point le rythme circadien et le découpage jour/nuit que le décompte des jours devient impossible. Le temps, dans le présent non borné de la narration, ne semble reprendre un cours normal que pour le pire. Pour signifier la progressive déshumanisation de William, d'abord. On perçoit clairement les différents états de sa malsaine amitié avec le gardien Arsenio et le progrès de son comportement sadique à l'égard du borgne Reyes. Pour marquer la débâcle ultime, ensuite, puisque le temps se remet en branle à l'arrivée de Francis, avec le développement brutal que l'on sait.

La sensation d'engourdissement temporel qui prévaut n'empêche pas la narration de s'inscrire dans une ligne diachronique. Bâti autour d'un événement premier, d'une intrigue et de son dénouement, le récit affiche en effet une architecture assez classique. Il arrive toutefois que le «je» qui articule le discours prenne le pas sur celui du personnage englué dans la diégèse, et provoque une sortie du temps linéaire, ou plutôt une fusion des plans. Ainsi, quelques jours après son entrée au boarding home, William reçoit la visite de son dernier et plus fidèle ami, El Negro. C'est la première fois, il convient de le préciser, que le narrateur fait référence à lui:

- ¿Qué hay de nuevo?-pregunto ansiosamente al Negro. El es mi contacto con la sociedad. Él va a reuniones de cubanos intelectuales, conversa de política, lee los periódicos, mira la televisión, y luego, cada una o dos semanas, viene a verme para transmitirme la esencia de sus correrías por el mundo (35).

Que se passe-t-il ici ? Le présent narratif qui jusque là avait œuvré à la mise en place du récit, c'est-à-dire à la présentation par William de son nouvel 
environnement, se mue insidieusement en un présent de répétition, qui garde sa validité pour le temps de l'élocution. Derrière ce «cada una o dos semanas viene a verme», c'est le présent du narrateur - et plus seulement celui du personnage - qui se dessine. Le plus souvent, le lecteur ne fait qu'avoir le soupçon de ce glissement du présent narratif au présent d'habitude, ou de leur co-présence, mais la stratification de ces temporalités dans la matière du présent verbal est absolument patente.

La prééminence du présent verbal est peut-être, au reste, l'expression la plus juste de la radicalité de l'échec qui frappe William. Si l'on se place du point de vue de l'élocution, il est indéniable que le présent de l'indicatif réactualise puissamment les situations décrites et en trahit la permanence. Le moment de l'énonciation est embrassé dans ce présent. Autrement dit, il n'est pas besoin d'attendre la fin du récit pour comprendre combien vaine est la tentative de William d'échapper au boarding home et au naufrage. Le présent verbal sonne depuis la première page comme une condamnation à perpétuité.

S'il est vrai qu'il n'est d'échec que conscient et accepté comme tel par qui l'endure, la lucidité de William, dans la déchéance, suffirait à nous le faire tenir pour le paradigme de la défaite. Et ce, dès les premières pages. Car on ne voit pas que le narrateur puisse tomber plus bas. Le discours de William et le temps verbal dominant convergent pour nous signifier 1. que l'arrivée au boarding home est bien une fin; 2. que c'est la station, et non le mouvement, qui régit le temps diégétique. L'éternité conjuguée au pire: voilà bien l'enfer. Cependant celui de Guillermo Rosales est disposé en épaisseurs successives, et, contrairement aux apparences, le personnage n'est pas encore au bout du chemin. Dans ce qu'on découvre être une traversée, un voyage, on ne touche véritablement les confins de l'enfer que lors du retour de William à l'asile, une fois ses projets réduits à néant.

L'auteur, donc, ne se satisfait pas du consentement du personnage à sa propre chute, il la redouble. Malgré l'avilissement, quelque chose était demeuré enfoui en lui de profondément humain, que l'arrivée de Francis met au jour: une tension irraisonnée vers l'avenir. Une des raisons pour lesquelles j'ai commencé par aborder La casa de los náufragos comme un texte de fiction tient précisément à l'équilibre de cette œuvre, qui s'organise, dans un mouvement de symétrie axiale, autour du pivot que constitue la survenue de Francis. Car la jeune femme, non contente d'interrompre le processus de déshumanisation du personnage, va le renverser. Il y a, en plus d'une cruauté consommée, un redoutable art de la construction romanesque 
dans ce procédé qui consiste à faire renaître William à la vie pour le mieux broyer.

A l'égal de tous les pensionnaires, Francis est réifiée par le regard qui est porté sur elle. Arsenio la qualifie de "nueva adquisición», et le comportement initial de William, la concernant, concorde avec l'attitude dont il est coutumier. Verrouillé à toute émotion, il agit à son encontre en prédateur sexuel. Francis va toutefois progressivement passer à ses yeux du statut d'objet à celui de sujet, ce dont attestent les choix qu'il opère dans la désignation: il la cantonne d'abord à sa folie - elle est «la loca»-, avant de se prendre d'affection pour elle - elle devient alors «la loquita» - et de la rendre, enfin, à l'humanité, en l'appelant par son nom. La découverte des aptitudes de dessinatrice de Francis est le facteur décisif de ce changement. Ce goût commun du Beau, qui prend chez William le tour de l'amour de la littérature, constitue en outre le premier signe d'une gémelléité confirmée par la parenté de leurs folies et la similitude de leur parcours vital.

A partir de la rencontre de Francis, la marche du temps se fait turbulente: dans cette nouvelle phase du récit, l'action est extraordinairement ramassée et contraste violemment avec l'impression de paralysie qui dominait jusque là. La relation amoureuse naissante va briser la dynamique implacable de l'échec. Le futur, à nouveau, fait sens. On peut facilement constater la magnitude du changement qui s'opère chez William en mettant en parallèle son sonore « ¡Vámonos del boarding home!» (72) avec un épisode antérieur, au cours duquel El Negro avait vainement tenté de dessiner un horizon à son désespoir, en suggérant inopinément un voyage en Espagne: «Willy -me dice-. ¡Vámonos de aquí!» (54). Tout au remâchement de sa déroute, le héros avait alors désamorcé la proposition par le rire.

La deuxième partie du roman préside à la mise en place d'un cercle vertueux. Là où William ne voyait qu'égoïsme et solitude, il découvre la solidarité des exilés. Il était apathique: il se montre désormais énergique et audacieux. Et au lieu que de brutaliser les fous, il les étreint. Plus surprenant encore, les voix qui l'assaillaient s'éteignent, entérinant le reflux de la folie. L'intrigue évolue tellement favorablement qu'on serait tenté de crier à la mièvrerie si l'on ne nourrissait la certitude d'un nouveau désastre. Rien de plus forcé que ce déroutement du récit, empêché de suivre sa pente naturelle vers le pire. Dans une torsion ultime, l'histoire reprendra finalement son cours logique avec l'arrestation de William, son séjour à l'hôpital, le départ forcé de Francis et le retour définitif du héros au boarding home. 
La mise en place du dénouement vaut qu'on s'y attache. A l'hôpital, dès avant la disparition de Francis, c'est-à-dire alors qu'il pourrait continuer de nourrir l'espoir d'une résolution favorable, William réendosse docilement les habits du vaincu. Il se retranche dans l'apathie - entendue au sens étymologique -, et cette vacance du sentiment est ce qui nous convainc le mieux de l'imminence d'une débâcle. Le coup d'arrêt mis à son projet semble avoir épuisé ses maigres capacités d'indignation. Or c'est l'humanité même de William qui était en jeu dans cette révolte insensée. Sa capitulation le replonge dans un abrutissement animal. Il semble donc que cet épisode n'ait eu d'autre dessein que de mettre en lumière une évidence: l'humanité tient toute dans la liberté et dans son exercice. Le propos ne diffère guère de celui du reste du roman: au long de cette parenthèse amoureuse, Guillermo Rosales ne fait que formuler ouvertement, dans le relief, ce qu'il exposait en creux lorsqu'il peignait les conséquences de l'aliénation sur William et ses compagnons d'infortune.

Le détachement de William se traduit en tout cas par une narration blanche, clinique - «factualiste», aurait dit William Burroughs. Jusqu'à ce qu'au fil de sa conversation avec un psychiatre bienveillant, le narrateur en revienne enfin aux récents événements. "Quise ser libre otra vez», dit-il, " quise huir del home donde vivía y empezar una nueva vida » (93). Le choix du temps verbal s'avère déterminant. A l'imparfait et à son déroulement dans la durée, William préfère le passé simple, qui dit la soudaineté de son espoir, mais aussi, du fait de l'absence de « déploiement» qui caractérise cette forme verbale (Barthes 1972: 26), la fragilité du changement qui s'est opéré à l'arrivée de Francis. L'imparfait n'épuise pas les alternatives à ce «quise». Car il n'est pas non plus un «he querido", et encore moins un "quiero » : le décrochement complet du présent qu'implique le passé simple est ici gros de sous-entendus. A quelques lignes de là, on découvre cette autre phrase: "Quizás no era todavía un gran amor», confie-t-il au Dr Paredes, "pero era algo que estaba floreciendo». Ces imparfaits, comme plus haut les passés simples, sanctionnent avec force le renoncement de William.

La défaite la plus radicale ne gît toutefois ni dans la déraison, ni dans l'écroulement du fragile édifice de sa reconstruction personnelle, mais bien dans la déshumanisation de William. Pour en cerner toutes les implications il faut revenir à la dichotomie vainqueurs / vaincus qui charpente le roman, et qui œuvre à plusieurs niveaux. La géographie du clivage vainqueurs / vaincus est matérialisée, en premier lieu, dans les espaces hermétiques du macrocosme et du microcosme. "Dehors et dedans», affirme Gaston Bachelard dans La 
poétique de l'espace, «forment une dialectique d'écartèlement et la géométrie évidente de cette dialectique nous aveugle dès que nous la faisons jouer dans des domaines métaphoriques. Elle a la netteté tranchante de la dialectique du oui et du non qui décide de tout» (Bachelard 1998: 191). Cette remarque s'applique parfaitement au roman, le porche du boarding home délimitant deux zones dont l'étanchéité n'est que rarement mise à l'épreuve. En résumé, donc: aux vainqueurs, la ville; aux vaincus, l'asile... Mais le principe dual régit également les relations au sein même de l'établissement. Or, dans cet espace, toute interprétation manichéenne est rendue impossible par la réversibilité des catégories: Arsenio, pathétique et cruel comparse de William, est un vaincu chez les vainqueurs et un vainqueur chez les vaincus. De la même façon, William, le vaincu par excellence, abuse sans vergogne de la faiblesse des pensionnaires du boarding home.

Ce qu'en réalité dévoile la double appartenance qui caractérise ces deux personnages, c'est la constance du rapport de force dans les relations humaines. La loi du plus fort s'applique uniformément dans et hors de l'asile, mais c'est au-dedans que cette réalité s'exprime avec la plus grande brutalité, du fait de la nouvelle déclinaison qu'y trouve le rapport vainqueur/ vaincu, désormais ramené à un tandem bourreau/victime. Cette flexion éclaire crûment le fait que les deux termes de la dichotomie initiale sont étroitement corrélés et que le vainqueur ne le devient pas indépendamment du vaincu: sa victoire se constitue sur les ruines d'une altérité qu'il lui faut bien souvent piétiner.

La typologie ne prend pas toujours un tour si élémentaire. Elle se trouve à un moment donné augmentée d'une catégorie médiane : «Este es mi fin. Yo, William Figueras, que [...] viví veinte años dentro de una revolución siendo victimario, testigo, víctima» (53). Si le protagoniste revient sur les rôles qu'il a successivement endossés à Cuba, c'est pour entériner le renversement de ce schéma initial en confrontant ce qu'il est devenu dans l'Ile - une victime - avec ce qu'il est en passe de devenir à l'asile - un bourreau. Arrivé en victime à l'hospice, William était passé, avant de s'adonner aux sulfureux plaisirs du sadisme, par une phase de latence et d'observation. Or, le pacte abominable que William scelle avec Arsenio en atteste, le témoin est partie prenante de ce qui se joue sous ses yeux. Silencieux, il rejoint déjà le camp des tortionnaires:

-Yo te veo a ti que le das un estrallón al viejo tuerto y me importa un carajo. Ahora, espero de ti lo mismo. Todo lo que tú veas que hago yo aquí, queda entre hombres. ¿Entendido? 
-Entendido -digo.

- ¿Mafia?

-Mafia -respondo $(42)^{4}$.

En somme, cette catégorie intermédiaire n'est qu'une coquille vide, et nous ramène à la dichotomie de départ. William découvre progressivement son aptitude à exercer le mal, sous l'œil bienveillant d'Arsenio. La culpabilité intermittente qu'il en retire (du moins au début) est à la mesure de son plaisir. C'est au reste à la survivance de ces scrupules que l'on reconnaît que William ne s'est pas encore dépouillé de son humanité. La jouissance sadique semble en tout cas la seule distraction possible à l'indifférence qui a gagné tout son être. Evoquant les tourments par lui infligés au borgne, il confesse: "Al recordarlo otra vez, mi cuerpo se estremece de placer» (38). Dans les violences faites à Reyes comme dans la pratique de la strangulation au cours de ses jeux avec Francis, le sadisme de William revêt une dimension orgastique. Et, pour perverse qu'elle soit, l'érotisation de la souffrance est à célébrer, en ce sens qu'elle relève de l'affect, et le maintient vivant.

Le véritable triomphe de la déshumanisation, donc, est postérieur, et concomitant de l'échec de la tentative de fuite. La défaite est autorisée avec indifférence par William lui-même, à son retour :

Curbelo se levanta y me empuja suavemente por los hombros.

-Ve-dice-. Come. En ningún lugar de este mundo estarás mejor que aquí.

Bajo la cabeza. Salgo, detrás de los locos, hacia el comedor (99).

Ce geste muet - non commenté - est suivi d'un silence typographique qui marque la transition vers un bref épilogue, au cours duquel la dégradation ultime prend la forme de la lassitude du sadique, qui lui fait appliquer mécaniquement ce qui auparavant était source de volupté:

En un rincón de la sala, Reyes, el tuerto, orina largamente. Arsenio llega hasta él y se quita el cinturón. Con la hebilla, da un violento cintarazo sobre la espalda del viejo tuerto. Llego hasta Arsenio y le quito el cinturón de las manos. Lo levanto sobre mi cabeza y lo dejo caer, con todas mis fuerzas, sobre el cuerpo esquelético del viejo tuerto (Rosales 2003: 100).

La conclusion du roman consacre donc la puissance de néantisation de ce lieu abject, érigé en tombeau de l'âme. Mais la narration froidement descriptive et le refus du commentaire, en cette fin de texte, ratifient aussi la perte de la parole, autrement radicale en ce qu'elle met en jeu bien autre chose que le sort du personnage: celui de l'auteur. Car si le texte accompagne

4 C’est moi qui souligne. 
le renoncement du personnage à l'humanité, il accompagne aussi celui de l'écrivain à l'écriture.

A ce stade de l'analyse, il importe d'éclairer le dialogue entre ce texte et la vie de Rosales, similaire à celle de son protagoniste jusque dans la mort qu'il lui pronostique: "Cojo una pistola imaginaria y me la llevo a la sien. Disparo» (57), lui fait-il dire. C’est précisément le geste qu'exécutera l'écrivain en 1993, cinq ans après la publication de Boarding home ${ }^{5}$. Sous le masque de William Figueras, donc, se dessine en filigrane le visage de Rosales. Cependant, faute d'une identité affichée entre le narrateur et l'auteur - faute de "pacte» (Lejeune 1975) - on ne saurait parler d'autobiographie. Tout au plus de roman autobiographique. L'appartenance au genre romanesque est de toute façon confirmée par certains éléments: la conduite du récit et l'aspect clos de l'œuvre, dont l'intrigue trouve sa résolution dans les limites du texte; ou encore la probable charge fictionnelle d'épisodes comme celui de l'intermède amoureux, si essentiel dans l'économie du récit.

Il n'y a nulle imposture dans cette opération de fictionnalisation de soi, dans cette projection de l'auteur sur un autre «je». Si Serge Doubrovsky disait de l'autofiction qu'elle consistait «pour capter le lecteur rétif», à «lui refiler sa vie réelle sous les espèces plus prestigieuses d'une existence imaginaire» (69), il faut peut-être plutôt voir dans le larvatus prodeo auquel se livre Guillermo Rosales une manière de distanciation d'avec sa propre déchéance.

La littérature avait échoué à éviter le naufrage à William, dont les lectures - depuis Ernest Hemingway, illustre suicidé et sujet à des troubles bipolaires, jusqu'à William Blake, écrivain tourmenté s'il en fût - n'étaient guère de nature à panser les plaies. Il faudrait ajouter à cela son impuissance à écrire. «Escribe algo » lui demande El Negro. «Trataré» (Rosales 2003: 54), répond-il. Mais il n'y a nulle mention, dans le roman, d'une tentative de cet ordre. Le labeur, le geste même de l'écriture en sont bannis. A la suite de William, Guillermo Rosales entre en 1987 dans le silence de l'écriture non sans avoir jeté à la face de ses contemporains ce texte désespéré, qui est tout de même quelque chose comme une sublimation. Carlos Victoria, membre de la génération de Mariel et frère de déroute de Guillermo Rosales et Reinaldo Arenas, porte témoignage de cette renonciation suprême de l'écrivain à l'écriture dans la nouvelle «La estrella fugaz», où il se met en

5 Tel est le titre que Rosales avait choisi de donner à ce texte, arbitrairement renommé par la maison d'édition. De quoi nourrir encore la thématique de la spoliation et de l'échec... 
scène en compagnie de ses amis disparus ${ }^{6}$. Le dialogue spéculaire y renvoie La casa de los náufragos à sa valeur testamentaire. Rosales, en effet, a détruit presque toute son œuvre, n'épargnant guère que les deux romans auxquels il est fait référence dans l'extrait qui suit, et derrière lesquels il faut reconnaître El juego de la viola et Boarding home. Il les juge avec la tranquille assurance de qui s'apprête à régler définitivement ses comptes avec la vie:

William sólo hablaba de su muerte inminente, que ocurriría esta tarde, o mañana, o a más tardar la semana que viene.

[...] - Antes de Navidad -decía William-. A los cuarenta y siete años.

Marcos asentía con la cabeza.

[...]-Lo que tienes que hacer es ponerte a escribir.

William daba una patada en el piso. Su carraspera se agravaba al gritar: - ¡No me hables de escribir! Ya yo escribí todo lo que tenía que escribir. Dos novelas - de pronto sonreía tenuemente y agregaba-. Excelentes, las dos. Como decía tu querido Keats: «Yo sé que mi nombre estará entre los poetas».

[...] William se olía las axilas, miraba a su alrededor y decía en voz baja: -Ellos piensan que soy un cobarde. Les voy a demostrar de lo que soy capaz. Tú mismo, aunque dices que sí, en el fondo no crees que yo pueda matarme.

-Ojalá que no lo hagas. Tienes todavía mucho que hacer.

-No tengo nada. Sólo hay algo que tengo que hacer (Victoria 1997).

Les lignes que Victoria consacre à Rosales sont un complément presque naturel au roman: le parcours de cet autre William prolonge et conclut, dans l'annonce du suicide, celui du héros de La casa de los náufragos. Grâce à quoi, dans «La estrella fugaz», s'accomplit la superposition définitive des images de William Figueras et Guillermo Rosales. C'est là la confirmation de ce que le manque à être du personnage de papier est abîmé dans un manque à être authentique, plus opaque et plus vaste, et qui ne relève décidément plus de l'analyse littéraire...

\section{Bibliographie}

Arenas, Reinaldo, 1996, Antes que anochezca, Barcelona, Tusquets, «Fábula». Bachelard, Gaston, 1998, La poétique de l'espace, Paris, PUF, «Quadrige».

6 Guillermo Rosales y prend aussi le nom de William. Cela n'a rien d'une coïncidence : c'est ainsi que le surnommaient ses proches. 
Barthes, Roland, 1972, Le degré zéro de l'écriture suivi de Nouveaux essais critiques, Paris, Seuil, «Points Esssais».

Doubrovsky, Serge, 1988, Autobiographiques, de Corneille à Sartre, Paris, PUF, «Perspectives critiques».

Lejeune, Philippe, 1975, Le pacte autobiographique, Paris, Seuil, «Poétique».

Rosales, Guillermo, 2003, La casa de los náufragos, Madrid, Siruela, «Libros del tiempo».

Victoria, Carlos, 1997, «La estrella fugaz», El resbaloso y otros cuentos, Miami, Universal, «Caniquí ». 